\title{
Artificial Intelligence of Things (AIoT) Technologies and Applications
}

\author{
Tien-Wen Sung $\left(\mathbb{D},{ }^{1}\right.$ Pei-Wei Tsai $\mathbb{D}^{2},{ }^{2}$ Tarek Gaber $\mathbb{D}^{3},{ }^{3}$ and Chao-Yang Lee $\mathbb{D}^{4}$ \\ ${ }^{1}$ Fujian Provincial Key Laboratory of Big Data Mining and Applications, Fujian University of Technology, Fuzhou, China \\ ${ }^{2}$ Department of Computer Science and Software Engineering, Swinburne University of Technology, Hawthorn, Australia \\ ${ }^{3}$ School of Science, Engineering \& Environment, University of Salford, Manchester, UK \\ ${ }^{4}$ Department of Aeronautical Engineering, National Formosa University, Yunlin, Taiwan
}

Correspondence should be addressed to Tien-Wen Sung; tienwen.sung@gmail.com

Received 19 July 2021; Accepted 19 July 2021; Published 15 August 2021

Copyright $\odot 2021$ Tien-Wen Sung et al. This is an open access article distributed under the Creative Commons Attribution License, which permits unrestricted use, distribution, and reproduction in any medium, provided the original work is properly cited.

AIoT (Artificial Intelligence of Things) is a relatively new term that has recently become a hot topic that combines two of the hottest acronyms, AI (Artificial Intelligence) and IoT (Internet of Things). IoT consists of interconnected things with built-in sensors and has the potential to generate or collect a vast amount of data. Individual IoT systems can be integrated into a large-scale system for various modern applications. With that comes a lot of collected or real-time data, an intelligent and efficient data processing is essential to make effective use of the information generated from these data. The data can be analyzed and utilized with AI for problem-solving or decision-making. Without AI, IoT would have limited value. AI can multiply the value of IoT; conversely, IoT can promote the learning and intelligence of AI. However, there are many challenges while deploying AIoT in practice. For instance, machine learning is one of the key technologies to be utilized in AIoT systems. Besides, there are many other issues such as complexity, efficiency, scalability, accuracy, and robustness related to the increasing modern AIoT systems and applications.

This Special Issue is aimed at publishing original and innovative research works that focus on challenging issues in the field of AIoT technology and applications. After the review process for evaluating all submitted manuscripts, there are nine papers accepted for publication in this special issue.

The paper titled "Semantic Integration of Sensor Knowledge on Artificial Internet of Things" by Y. Huang et al. describes the problem of data meaning matching in cooperations among heterogeneous sensor-based AIoT systems. The authors propose an ontology-based approach to deal with the semantic meaning matching problem and propose a compact Particle Swarm Optimization (cPSO) algorithm to improve the quality of ontology alignment in different sensor ontologies. The experiment results indicate that the proposed approach statistically outperforms other state-of-the-art sensor ontology matching techniques.

The paper titled "Quasiconformal Mapping Kernel Machine Learning-Based Intelligent Hyperspectral Data Classification for Internet Information Retrieval" by J. Liu and Y. Qiao focuses on the proposed intelligent data classification algorithm based on machine learning. The approach of quasiconformal kernel mapping learning with Mahalanobis distance kernel functions is presented with a clear framework. The algorithm can be utilized for Internet-based hyperspectral data retrieval, which is important for many AIoT applications such as image and video-based ones. In the experiments, it achieves advantages on large training sample construction and is effective to image data classification.

The paper titled "Flower End-to-End Detection Based on YOLOv4 Using a Mobile Device" by Z. Cheng and F. Zhang proposes a flower detection approach for smart garden applications. The method of anchor-based end-to-end deep convolutional neural network with YOLO is presented. With the designed architecture of the flower detection system, the proposed method performs an improved detection speed while the accuracy is similar to that of the other methods.

The paper titled "A Node Location Method in Wireless Sensor Networks Based on a Hybrid Optimization Algorithm" 
by J.-S. Pan et al. deals with the sensor node positioning problem and proposes a new algorithm named WOA-QT that combines the Whale Optimization Algorithm (WOA) with QUasiAffine Transformation Evolutionary (QUATRE) algorithm. The algorithm optimizes the received signal strength indication (RSSI) ranging and weighted centroid positioning (WCL) for improving the positioning accuracy. In the paper, 30 benchmark functions were used to evaluate the performance. The proposed method achieves a satisfactory positioning accuracy.

The paper titled "URDNet: A Unified Regression Network for GGO Detection in Lung CT Images" by W. Liu et al. describes the topic of ground-glass opacity (GGO) nodule detection in images for medical IoT systems. The authors propose an end-to-end deep convolutional neural network with a multi-input and multioutput structure. A two-stage training method is used, which includes the network backbone training and URDNet fine-tuning with pretrained weights. The LIDCIDRI dataset is used to evaluate the performance, and the proposed method achieves a sensitivity of $90.8 \%$. The approach could offer a useful tool in medical applications.

The paper titled "High-Dimensional Text Clustering by Dimensionality Reduction and Improved Density Peak" by Y. Sun and J. Platoš focuses on the intelligent text-based data clustering technique. The authors propose a Stacked Random Projection (SRP) method to reduce the dimensionality of high-dimensional text data. An enhanced algorithm named DPC-K-means based on density peak clustering algorithm is also proposed. Seven text datasets, including BBC News and Amazon product reviews, were used to validate the proposed approach. The experiment results indicate the method is superior to other compared algorithms.

The paper titled "Applying Robust Intelligent Algorithm and Internet of Things to Global Maximum Power Point Tracking of Solar Photovoltaic Systems" by E.-C. Chang proposes an IoT-based model and control approach to monitor solar photovoltaic (PV) systems and ensure maximum power point tracking. A neural network based on quantum particle swarm optimization (QPSO) and radial basis function (RBF) is proposed. It is used to find the maximum power of the photovoltaic array and maintain the highest PV energy conversion efficiency. The mathematical analysis and simulations show the achievement of tracking accuracy and robust adaptation.

The paper titled "Developing Deep Survival Model for Remaining Useful Life Estimation Based on Convolutional and Long Short-Term Memory Neural Networks" by C.-H. $\mathrm{Chu}$ et al. focuses on the remaining useful life estimation and failure probability of machines for smart manufacturing. The authors propose an integrated deep learning approach with the convolutional neural network (CNN) and long short-term memory (LSTM) network to deal with the problem. The dataset provided by NASA is utilized to evaluate the performance. The results indicate that the proposed model can capture the degradation trend of a fault under complex conditions and avoid failure with the early prediction.

The paper titled "Fuzzy Obstacle Avoidance for the Mobile System of Service Robots" by S.-P. Tseng et al. designs and implements a service robot featured by obstacle avoidance and target user tracking. The obstacle avoidance is achieved by several ultrasonic sensors and a fuzzy-based obstacle detection method. The proposed method can effectively improve the anomaly detection of sensors when facing extreme conditions. It has low computational complexity and can facilitate real-time operation in the system. The experiment was performed in a real indoor environment, and an outstanding result was achieved.

Tien-Wen Sung
Pei-Wei Tsai
Tarek Gaber
Chao-Yang Lee

\section{Conflicts of Interest}

The guest editors declare that they have no conflicts of interest regarding the publication of this special issue. 